\title{
Cause of death for elders with colorectal cancer: a real-world data analysis
}

\author{
Renjie Wang ${ }^{1,2 \#}$, Lingyu Han ${ }^{1,2 \#}$, Weixing Dai ${ }^{1,2}$, Shaobo Mo ${ }^{1,2}$, Wenqiang Xiang ${ }^{1,2}$, Qingguo $\mathrm{Li}^{1,2}, \mathrm{Ye} \mathrm{Xu}^{1,2}$, \\ Guoxiang Cai ${ }^{1,2}$
}

${ }^{1}$ Department of Colorectal Surgery, Fudan University Shanghai Cancer Center, Shanghai 200032, China; ${ }^{2}$ Department of Oncology, Shanghai Medical College, Fudan University, Shanghai 200032, China

Contributions: (I) Conception and design: R Wang, L Han, G Cai; (II) Administrative support: R Wang, W Dai, S Mo, W Xiang, Q Li; (III) Provision of study materials or patients: L Han, G Cai, Y Xu, W Xiang; (IV) Collection and assembly of data: R Wang, L Han, S Mo, Q Li; (V) Data analysis and interpretation: R Wang, W Dai, L Han, Y Xu; (VI) Manuscript writing: All authors; (VII) Final approval of manuscript: All authors.

\#These authors contributed equally to this work as co-first authors.

Correspondence to: Guoxiang Cai, MD, PhD. Department of Colorectal Surgery, Fudan University Shanghai Cancer Center, Department of Oncology, Shanghai Medical College, Fudan University, 270 Dong'an Road, Shanghai 200032, China. Email: gxcaifuscc@163.com.

\begin{abstract}
Background: Many patients surviving from colorectal cancer die of causes irrelevant to cancer. This study was designed to describe the leading causes of death among older patients diagnosed with colorectal cancer and assess factors that are related to colorectal cancer mortality versus mortality from other causes.

Methods: Patients over 65-year-old diagnosed with colorectal cancer between 2000 and 2014 were extracted from Surveillance, Epidemiology, and End Results (SEER) linked database.

Results: A total of 136,872 patients with colorectal cancer met the inclusion criteria. The median followup time was approximately 3 years. Forty-five point seven percent of them were alive at the end of followup, and colorectal cancer still accounted for the most cases of deaths. However, patients with tumor of Grade I or TNM stage I-II were more likely to die from other causes. The fully-adjusted relative hazards ratio (HR) shows age, gender, tumor site, chemotherapy, tumor characteristics of grade and TNM stage affected both types of mortality. Race only affected mortality of other causes. Cardiovascular disease (CVD) was a noticeable cause of death among patients with stage I colorectal cancer. With longer follow-up, deaths due to colorectal cancer decreased while deaths of CVD, pulmonary diseases and other cause of death increased.

Conclusions: Colorectal cancer still accounts for most deaths in elderly patients. However, comorbidities including CVD/COPD were associated with the deaths of colorectal cancer patients over 65 . As survival time increases, comorbidities should be considered for cancer treatment. Management of CVD/COPD among elderly patients can help improve overall survival (OS) in colorectal cancer.
\end{abstract}

Keywords: Cause of death; colorectal cancer; Surveillance, Epidemiology, and End Results (SEER); elder

Submitted Dec 06, 2019. Accepted for publication Feb 14, 2020.

doi: 10.21037/jgo.2020.03.04

View this article at: http://dx.doi.org/10.21037/jgo.2020.03.04

\section{Introduction}

According to GLOBOCAN 2018, colorectal cancer was listed as $3^{\text {rd }}$ most common cancer $(6.1 \%)$, and $2^{\text {nd }}$ leading cause of cancer death $(9.2 \%)$ in both sexes combined (1). However, death rates of colorectal cancer declined by approximately $2 \%$ per year in the 1990 s and by approximately $3 \%$ per year in the last decade (2). This is due to the improving clinical practice in cancer treatment and management in developed countries. Also, development and popularization of screening and early detection programs have profound impact (3). With more precancerous lesions found and treated under colonoscopy, more early tumors have been detected and received radical resections. As a result, the colorectal cancer prevention, treatment and surveillance 
are constantly changing. More targeted plans can reduce labor costs and financial input, and get more returns $(4,5)$. However, despite of the cancer treatment, the survivorship of colorectal cancer patients also depends on many other factors, including age at diagnosis, comorbidities and society connections. Previous studies have shown that preexisting comorbidities had specific impact on survival of patients with colorectal cancer $(6,7)$, and more than one-third of deaths would occur in individuals over 80 -year-old (2). The current study is designed to find the causes of death for elderly patients with colorectal cancer and assess factors on colorectal cancer mortality and mortality of other causes among colorectal cancer patients aged over 65 .

Conducting epidemiologic research on cancer survivorship is based on all-cause mortality, which means the most commonly used outcome in clinical studies for colorectal cancer is the sum of colorectal cancer-specific mortality and mortality of other causes. Assessment of outcomes for both colorectal cancer-specific mortality and mortality of other causes can assist doctors in finding prognostic indicators and picking out the most important medical care to improve patients' survival. This study exhibited features of colorectal cancer-specific mortality and other mortality and explored the leading cause of death among colorectal cancer patients from the Surveillance, Epidemiology, and End Results (SEER) database.

\section{Methods}

\section{Patient selection}

Data of the current study on the patients with colorectal cancer between 2000 and 2014 was retrieved from the SEER database. Sponsored by the National Cancer Institute, the SEER database covers $26 \%$ population from 18 cancer registries of USA with both incidence and survival information of malignancies.

Information of both treatment details and clinicopathological factors were extracted. Patients over 65 years old who met the following criteria were included: (I) patients were pathologically diagnosed with colorectal cancer; (II) colorectal cancer was the only primary carcinoma. Patients with incomplete TNM staging or survival data were excluded.

\section{Statistical analyze}

The study cohorts were divided into three subgroups:
(I) patients who were alive during our study period; (II) patients who died as a result of colorectal cancer-specific causes at the end of the study period; (III) patients who died of other causes. For further analysis of other-causes of death, we categorized other-cause mortality into three groups: the cardiovascular disease (CVD), pulmonary diseases including chronic obstructive pulmonary disease (COPD) and others.

For depicting the clinicopathological characteristics of cohorts, quantitative values and medians with interquartile ranges (IQRs) were utilized. Multivariate Cox proportional hazard regression models were constructed after univariate Cox proportional hazard regression models to determine the association between characteristic factors and survival status. Survival curves of 4 groups with different age ranges were drawn using the Kaplan-Meier method. Differences in survival were examined by the Log-rank test. Tumor stage was coded based on the UICC/AJCC TNM staging system (8th edition). The cutoff points based on age at diagnosis sprang from previous study (8).

$\mathrm{R}$ software for Windows (version R-3.4.3, the $\mathrm{R}$ Foundation for statistical computing) was performed for all statistical analyses. All statistical comparisons were two sided. And a $\mathrm{P}<0.05$ was assessed as a threshold of statistical significance.

\section{Results}

A total of 136,872 patients who met the inclusion criteria were included in our cohorts. Among these patients, 74,307 individuals (54.3\%) died during the study period: 45,131 (33.0\%) of the study cohorts died due to colorectal cancer, while the other $29,176(21.3 \%)$ died as a result of other causes. Among them, 14,489 patients died of CVD and 2,413 died of COPD or other pulmonary diseases. The median follow-up time was 37 months (IQR: 13-74), and the median age at death was 79 years old (IQR: 72-84). Generally, colorectal cancer still accounted for the most cases of deaths while patients with tumor of Grade I or TNM stage I-II were more likely to die from non-tumorous causes (Table 1).

Both the age-adjusted relative hazards ratio (HR) of death induced by colorectal cancer and other causes were greater among patients with advanced age, male patients, treatment with chemoradiotherapy and higher TNM stage (Table 2). After adjustment for age, TNM stage, histopathologic grade, tumor site and therapeutic schemes, HR of colorectal cancer-specific mortality was different 
Table 1 Clinicopathological characteristics in three subgroups of mortality status in colorectal cancer patients over 65-year-old in SEER database

\begin{tabular}{|c|c|c|c|c|c|c|}
\hline Variable & \multicolumn{2}{|c|}{ Alive } & \multicolumn{2}{|c|}{ Colorectal cancer deaths } & \multicolumn{2}{|c|}{ Other-cause deaths } \\
\hline \multicolumn{7}{|l|}{ Age } \\
\hline $65-75$ & 36,037 & 26.33 & 17,472 & 12.77 & 7,366 & 5.38 \\
\hline $75-85$ & 21,098 & 15.41 & 17,739 & 12.96 & 13,322 & 9.73 \\
\hline \multicolumn{7}{|l|}{ Gender } \\
\hline Female & 33,523 & 24.49 & 24,012 & 17.54 & 15,273 & 11.16 \\
\hline Male & 29,042 & 21.22 & 21,119 & 15.43 & 13,903 & 10.16 \\
\hline \multicolumn{7}{|c|}{ Race/ethnicity } \\
\hline Other & 6,446 & 4.71 & 3,400 & 2.48 & 1,695 & 1.24 \\
\hline Unknown & 498 & 0.36 & 43 & 0.03 & 18 & 0.01 \\
\hline \multicolumn{7}{|l|}{ Site } \\
\hline Right & 31,557 & 23.06 & 22,572 & 16.49 & 15,823 & 11.56 \\
\hline Left & 31,008 & 22.65 & 22,559 & 16.48 & 13,353 & 9.76 \\
\hline \multicolumn{7}{|c|}{ Chemoradiotherapy } \\
\hline No & 43,683 & 31.92 & 28,043 & 20.49 & 24,710 & 18.05 \\
\hline IV & 1,190 & 0.87 & 1,478 & 1.08 & 529 & 0.39 \\
\hline Unknown & 3,728 & 2.72 & 4,761 & 3.48 & 1,900 & 1.39 \\
\hline \multicolumn{7}{|l|}{ TNM stage } \\
\hline 1 & 17,386 & 12.70 & 2,078 & 1.52 & 6,994 & 5.11 \\
\hline II & 21,957 & 16.04 & 7,851 & 5.74 & 10,354 & 7.56 \\
\hline III & 20,884 & 15.26 & 17,870 & 13.06 & 10,264 & 7.50 \\
\hline IV & 2,338 & 1.71 & 17,332 & 12.66 & 1,564 & 1.14 \\
\hline
\end{tabular}

from mortality of other-causes. Age, gender, tumor site, chemotherapy, tumor characteristics of grade and TNM stage affected both types of mortality, while race only affected mortality of other-causes.

The proportion of different causes of death was various by age and tumor stages (Figure 1). Patients suffered from tumor with higher stages were much more likely to die as a result of colorectal cancer instead of other causes. On the other hand, other causes of death happened more likely among patients with stage I-II cancers. In subgroups 
Table 2 Age-adjusted and multivariate analysis of colorectal cancer mortality and other-cause mortality in patients over 65-year-old in SEER database

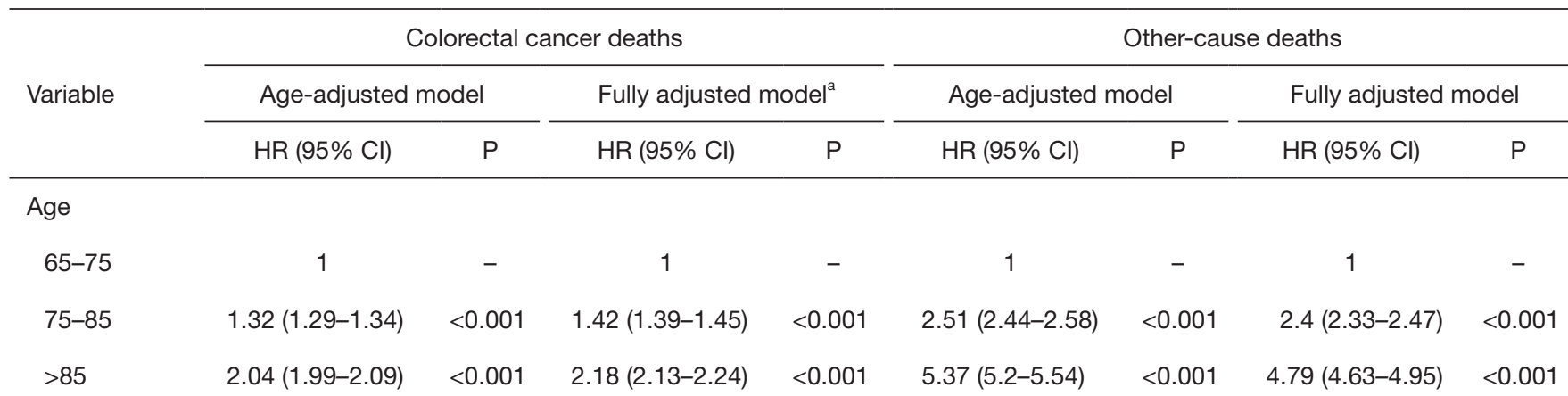

Gender

Female

Male

$1.09(1.07-1.11)$

$<0.001$

\section{1}

Race/ethnicity

Black

Other

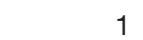

Unknown

$0.7(0.67-0.73)$

$0.19(0.14-0.25)$

$0.78(0.76-0.8)$

White

Site

Right

Left

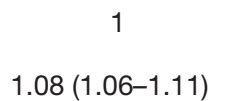

$-$

$<0.001$

$1.08(1.06-1.1)$

$-$

$<0.001$

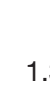

1.31

1

$18-1.34)$

$-$

$<0.001$

$1.34(1.31-1.38) \quad<0.001$

Chemoradio-therapy

No

Yes

$1.63(1.6-1.67)$

Grade$$
\text { । }
$$

II

III

IV

Unknown

TNM stage

\begin{tabular}{lccccccccc} 
I & 1 & - & 1 & - & 1 & - & 1 & - \\
II & $2.64(2.51-2.77)$ & $<0.001$ & $2.48(2.36-2.6)$ & $<0.001$ & $1.05(1.02-1.08)$ & 0.002 & $1.11(1.08-1.15)$ & $<0.001$ \\
III & $5.98(5.72-6.26)$ & $<0.001$ & $5.98(5.71-6.27)$ & $<0.001$ & $1.13(1.1-1.16)$ & $<0.001$ & $1.38(1.34-1.43)$ & $<0.001$ \\
IV & $27.27(26.04-28.56)$ & $<0.001$ & $27.38(26.09-28.73)$ & $<0.001$ & $1.05(0.99-1.11)$ & 0.088 & $1.39(1.31-1.48)$ & $<0.001$ \\
\hline
\end{tabular}

\footnotetext{
${ }^{\mathrm{a}}$, multivariate analysis including age, gender, race, histological grade, TNM-stage and adjuvant therapy.
} 


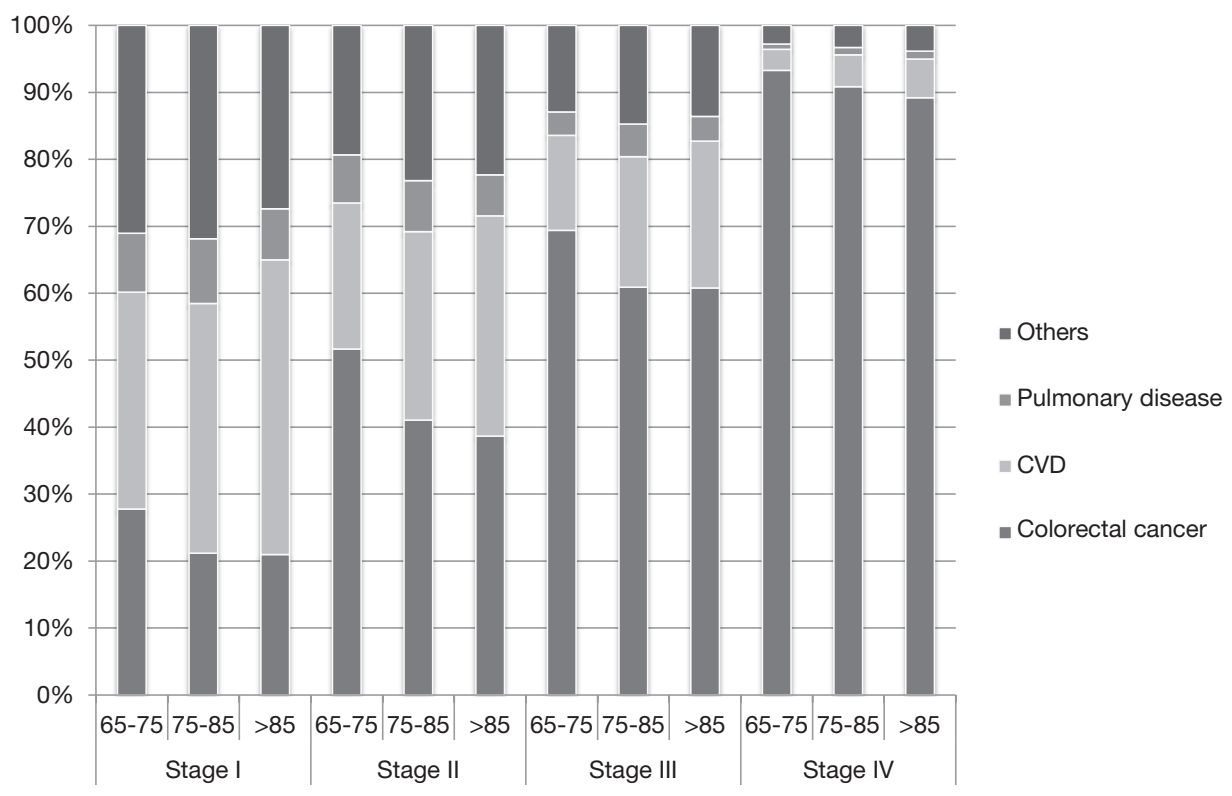

Figure 1 Proportion of different leading causes of death in colorectal cancer patients over 65 of subgroups divided by age at diagnosis and by stage of disease.

of different age ranges, CVD accounted for the highest proportion of deaths among patients diagnosed with stage I colorectal cancer. CVD was also the second leading cause of deaths for patients with stage II-IV colorectal cancer. Within all stages, there was increasing proportion of othercauses deaths as patients aged.

The proportion of cumulative causes of death was also dependent on the length of follow-up time. With longer follow-up, the proportion of deaths due to colorectal cancer decreased while deaths of CVD, pulmonary diseases and other causes of death increased continuously (Figure 2). The other causes of death in this category included deaths caused by Alzheimer's disease ( $\mathrm{n}=1,498,1.09 \%)$ and diabetes $(\mathrm{n}=1,081,0.79 \%)$.

\section{Discussion}

In our clinical practice, more and more elderly patients with colorectal cancer receive surgery and/or chemoradiotherapy. In the meantime, they are more likely to have comorbidities, such as CVD, pulmonary disease (such as COPD and influenza), making treatment riskier (9). According to scientific statistics, age is an independent prognostic factor for both in-hospital morbidity and mortality after colorectal surgery $(10,11)$. As a result, we always hold a negative attitude towards the treatment of colorectal cancer in elderly patients (12). In clinical practice, we prefer give up surgery or decrease chemotherapy dose, because we have a traditional idea that elderly patients may not die from colorectal cancer but from comorbidities. However, in our cohort, we found only patients with early grade tumor, and early stage were more likely to die as a result of other causes, while colorectal cancer still accounted for most deaths in elderly patients. Therefore, we advocate active anti-tumor therapy for elderly patients with colorectal cancer.

Patients with early grade tumor and early stage were more likely to die as a result of other causes. For these patients, in addition to anti-tumor therapy, we should put more attention on diseases of other causes, such as CVD, COPD and other lung diseases. In our study, CVD was exhibited as the second leading cause of death in our cohorts ( $n=14,489,10.59 \%)$, second only to colorectal cancer $(n=45,131,32.97 \%)$. And especially in patients with stage I colorectal cancer, CVD was the primary cause of death. This is because patients over 65 have a higher incidence of comorbidities and patients with comorbidities are more likely to die as a result of other causes. When patients survive longer, CVD may become the leading cause of death.

In both HR of colorectal cancer mortality and othercause mortality, men are significantly more at risk than 


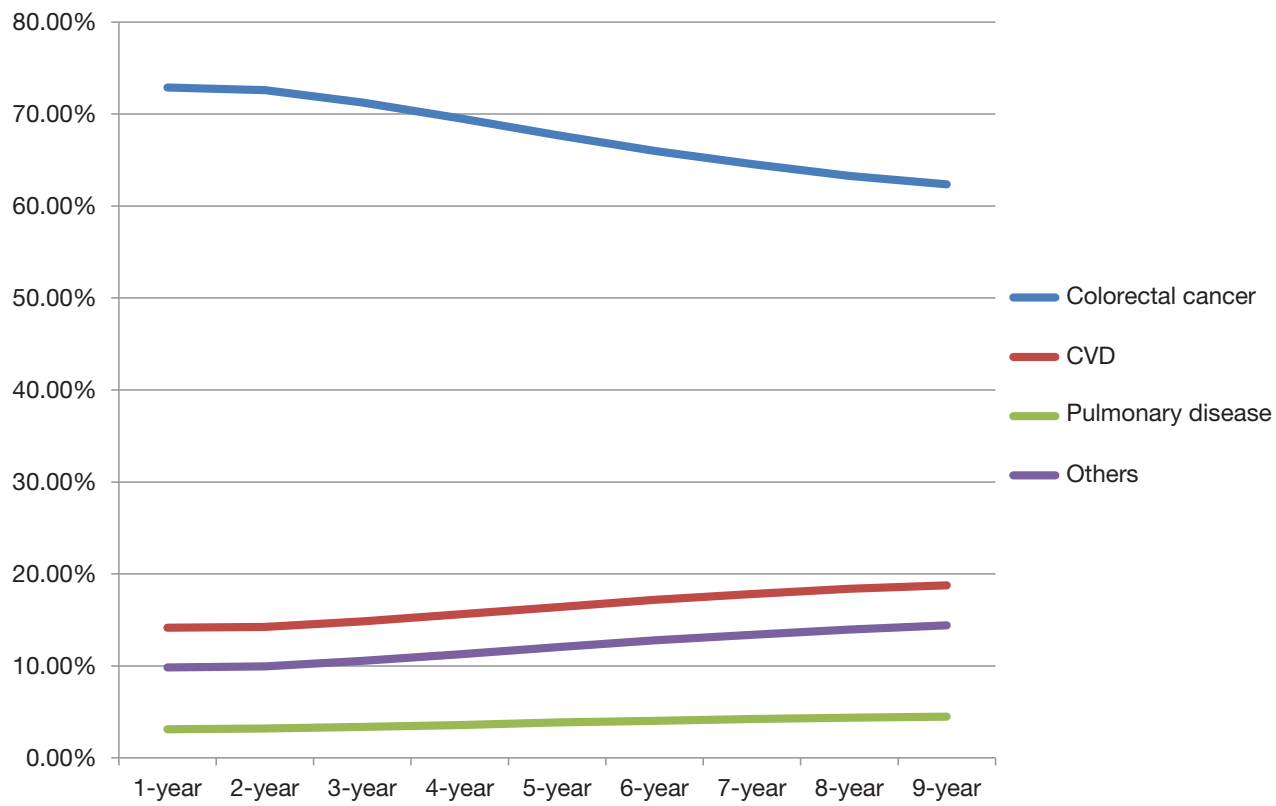

Figure 2 Changing distribution of cumulative causes of death by time since colorectal cancer diagnosis.

women. This can be explained by several reasons. First, estrogen has a protective effect on colorectal cancer in women, and women have a 7-8 years lag in developing colorectal cancer (13). Second, when it comes to lifestyle and health, women are undoubtedly superior to men in general. Smoking and drinking are still bad habits that many male patients with colorectal cancer can't get rid of. Third, in both east and west, men are still generally given more responsibility than women. Together with the society's 'definition' of male roles, men still tend to be more aggressive and endure problems even if they cannot hold on. These are not only bad for their physical and mental health, but also bad for early detection and diagnosis.

Ethnic differences also often affect treatment decision. Generally, white patients have relative low hazards of colorectal cancer deaths than black patients. However, for other causes of deaths, there was no statistical difference between these two races. Since colorectal cancer incidence and mortality are declining over the past decades owing to the adoption of effective screening programs (14), we call for more attention to colorectal cancer screening in black people so as to eliminate the difference in mortality.

In terms of tumor site, we can see that the survival of left colon cancer is slightly worse than that of right colon cancer in terms of both colorectal cancer death and other-causes death. This is contrary to other literature, which advocates that due to differences in embryo origin, anatomical structure and physiological function, right colon cancer may have worse biological behavior (15). However, other studies also have shown no significant difference in overall survival (OS) and disease-free survival (DFS) after surgery for left and right colon cancer, and the OS and DFS for early right colon cancer are higher than those for left colon cancer (15). Since stage IV patients accounted for only a small portion of our study cohort, it is reasonable to believe that the survival of patients with right colon cancer may be better than those with left colon cancer.

In HR of colorectal cancer mortality, we found that in age-adjusted model, chemoradiotherapy is a risk factor for colorectal cancer death. However, after fully adjusted by age, tumor stage, grade, site and treatments, chemoradiotherapy becomes a protective factor for colorectal cancer death. This can be explained by the fact that for all patients, chemoradiotherapy often involves patients with late stage and poorly differentiated tumors, so chemoradiotherapy is a risk factor; but after adjusted by all factors, for patients with the same stage and characteristics, chemoradiotherapy offers a better survival than denying it.

Tumor grade is a risk factor for colorectal cancer death, but not influence the other cause of death. However, TNM staging is a risk factor for both colorectal cancer death and other cause of death. This is because the severity of colorectal cancer will also affect non-neoplastic diseases, especially for patients with metastasis who 
need intense chemoradiotherapy, etc. The side effects of chemoradiotherapy will also increase the prevalence of comorbidities.

Of course, our study is more or less imperfect, which is limited by the validity of death coding. The validity of cause-of-death confirmation is not entirely accurate, which has been found to vary in different cancer site, time of diagnosis and ages at death (16). Also, the Framingham Heart Study (17) have shown that heart disease may be over-evaluated in objective diagnosis for the cause of death generally. So, the rates of CVD may be overestimated in this study population diagnosed with colorectal cancer. In addition, there are a number of inevitably missing records for race, grade and surgery status. This information may influence the conclusion of our study. In conclusion, colorectal cancer still accounts for most deaths in elderly patients. However, elderly patients are associated with increasing risk of mortality from other comorbidities, especially CVD and pulmonary diseases. Patients with Grade I tumor and TNM stage I-II were more likely to die as a result of other causes rather than colorectal cancer. As survival time increases, deaths due to colorectal cancer decrease while deaths of CVD, pulmonary diseases increase. As a result, we should still launch active anti-tumor therapy for elderly patients with colorectal cancer, but for early stage tumors and as patients live longer, treatment of other existing comorbidities should also be considered into the schedule of patients' management. Especially among colorectal cancer survivors over 65, proper management of CVD/COPD may improve patients' OS.

\section{Acknowledgments}

Funding: None.

\section{Footnote}

Conflicts of Interest: All authors have completed the ICMJE uniform disclosure form (available at http://dx.doi. org/10.21037/jgo.2020.03.04). The authors have no conflicts of interest to declare.

Ethical Statement: The authors are accountable for all aspects of the work in ensuring that questions related to the accuracy or integrity of any part of the work are appropriately investigated and resolved.

Open Access Statement: This is an Open Access article distributed in accordance with the Creative Commons Attribution-NonCommercial-NoDerivs 4.0 International License (CC BY-NC-ND 4.0), which permits the noncommercial replication and distribution of the article with the strict proviso that no changes or edits are made and the original work is properly cited (including links to both the formal publication through the relevant DOI and the license). See: https://creativecommons.org/licenses/by-nc$\mathrm{nd} / 4.0 \%$.

\section{References}

1. Bray F, Ferlay J, Soerjomataram I, et al. Global cancer statistics 2018: GLOBOCAN estimates of incidence and mortality worldwide for 36 cancers in 185 countries. CA Cancer J Clin 2018;68:394-424.

2. Siegel R, Desantis C, Jemal A. Colorectal cancer statistics, 2014. CA Cancer J Clin 2014;64:104-17.

3. Smith RA, Andrews KS, Brooks D, et al. Cancer screening in the United States, 2018: A review of current American Cancer Society guidelines and current issues in cancer screening. CA Cancer J Clin 2018;68:297-316.

4. Dhaliwal A, Vlachostergios PJ, Oikonomou KG, et al. Fecal DNA testing for colorectal cancer screening: Molecular targets and perspectives. World J Gastrointest Oncol 2015;7:178-83.

5. Brenner H, Stock C, Hoffmeister M. Effect of screening sigmoidoscopy and screening colonoscopy on colorectal cancer incidence and mortality: systematic review and meta-analysis of randomised controlled trials and observational studies. BMJ 2014;348:g2467.

6. Boakye D, Rillmann B, Walter V, et al. Impact of comorbidity and frailty on prognosis in colorectal cancer patients: A systematic review and meta-analysis. Cancer Treat Rev 2018;64:30-9.

7. Edwards BK, Noone AM, Mariotto AB, et al. Annual Report to the Nation on the status of cancer, 1975-2010, featuring prevalence of comorbidity and impact on survival among persons with lung, colorectal, breast, or prostate cancer. Cancer 2014;120:1290-314.

8. Patnaik JL, Byers T, DiGuiseppi C, et al. Cardiovascular disease competes with breast cancer as the leading cause of death for older females diagnosed with breast cancer: a retrospective cohort study. Breast Cancer Res 2011;13:R64.

9. Wang JW, Sun L, Ding N, et al. The association between comorbidities and the quality of life among colorectal cancer survivors in the People's Republic of China. Patient 
Prefer Adherence 2016;10:1071-7.

10. Jafari MD, Jafari F, Halabi WJ, et al. Colorectal Cancer Resections in the Aging US Population: A Trend Toward Decreasing Rates and Improved Outcomes. JAMA Surg 2014;149:557-64.

11. Turrentine FE, Wang H, Simpson VB, et al. Surgical risk factors, morbidity, and mortality in elderly patients. J Am Coll Surg 2006;203:865-77.

12. Yamano T, Yamauchi S, Kimura K, et al. Influence of age and comorbidity on prognosis and application of adjuvant chemotherapy in elderly Japanese patients with colorectal cancer: A retrospective multicentre study. Eur J Cancer 2017;81:90-101.

13. Williams C, DiLeo A, Niv Y, et al. Estrogen receptor beta as target for colorectal cancer prevention. Cancer Lett

Cite this article as: Wang R, Han L, Dai W, Mo S, Xiang W, Li Q, Xu Y, Cai G. Cause of death for elders with colorectal cancer: a real-world data analysis. J Gastrointest Oncol 2020;11(2):269-276. doi: 10.21037/jgo.2020.03.04
2016;372:48-56.

14. Itatani Y, Kawada K, Sakai Y. Treatment of Elderly Patients with Colorectal Cancer. Biomed Res Int 2018;2018:2176056.

15. Petrelli F, Tomasello G, Borgonovo K, et al. Prognostic Survival Associated With Left-Sided vs Right-Sided Colon Cancer: A Systematic Review and Meta-analysis. JAMA Oncol 2017;3:211-9.

16. Hoel DG, Ron E, Carter R, et al. Influence of death certificate errors on cancer mortality trends. J Natl Cancer Inst 1993;85:1063-8.

17. Lloyd-Jones DM, Martin DO, Larson MG, et al. Accuracy of death certificates for coding coronary heart disease as the cause of death. Ann Intern Med 1998;129:1020-6. 